\title{
Simulation of Radar Reflectivity and Surface Measurements of Rainfall
}

\author{
V. Chandrasekar AND V. N. BRingi \\ Department of Electrical Engineering, Colorado State University, Fort Collins, CO 80523
}

(Manuscript received 16 October 1986, in final form 12 March 1987)

\section{ABSTRACT}

\begin{abstract}
Raindrop size distributions (RSDs) are often estimated using surface raindrop sampling devices (e.g., disdrometers) or optical array (2D-PMS) probes. A number of authors have used these measured distributions to compute certain higher-order RSD moments that correspond to radar reflectivity, attenuation, optical extinction, etc. Scatter plots of these RSD moments versus disdrometer-measured rainrates are then used to deduce physical relationships between radar reflectivity, attenuation, etc., which are measured by independent instruments (e.g., radar), and rainrate. In this paper we simulate RSDs of the gamma form as well as radar reflectivity (via time series simulation) to study the correlation structure of radar estimates versus rainrate as opposed to RSD moment estimates versus rainrate. Simulations offer a powerful method of studying the statistics of radar and surface RSD measurements since the "natural" RSD fluctuations can be introduced separately. In our simulations we vary the parameters $N_{0}, D_{0}$ and $m$ of a gamma distribution over the range normally found in rainfall, as well as varying the device sampling volume. We apply our simulations to explain some possible features related to discrepancies which can arise when radar rainfall measurements are compared with surface or aircraft-based sampling devices.
\end{abstract}

\section{Introduction}

The radar remote measurement of rain intensity is a problem of continuing interest to radar meteorologists. For example, the remote monitoring of flash-flood producing storms is an important applications area. Even though considerable progress has been made in the development of new radar measurement techniques, e.g., using dual-polarized, dual-frequency or differential phase shift, Seliga and Bringi $(1976,1978)$, Goddard and Cherry (1984), Sachidananda and Zrnic (1986), Jameson (1985), Mueller (1984), the problems associated with the error structure of remotely sensed precipitation estimates appear to have received little attention so far in precipitation research, AGU (1984).

A pulsed, meteorological radar illuminates a radar resolution volume which depends on range, antenna pattern and pulse width; typically the volume is approximately $0.1 \mathrm{~km}^{3}$ for a range of $50 \mathrm{~km}, 1^{\circ}$ beam width and $1 \mu \mathrm{sec}$ pulse, Doviak and Zrnic (1984). Within this volume, hydrometeors are assumed to be randomly positioned, and constitute a random medium from. which radar measurements are obtained. Furthermore, the fractional volume concentration of the scatterers is generally very small $(\ll 1 \%)$ so that the independent backscatter approximation is valid. Hence, the average backscattered power (or reflectivity) is proportional to the (incoherent) sum of powers backscattered by each particle within the resolution volume. Statistical fluctuations of the received power are related to the Doppler velocity spectrum of the particles within the resolution volume. Conventional Doppler radars measure the mean power (or reflectivity, $Z$ ) as well as the first moment of the Doppler spectrum, i.e., the mean velocity. Rainfall rate, or the vertical flux of raindrops contained within the resolution volume, is conventionally related to $Z$ by power law equations of the form $Z=a R^{b}$, where $a$ and $b$ depend on the unknown raindrop size distribution (RSD), Atlas (1964), Ulbrich (1983). The radar measured mean power $(\bar{P})$, an electromagnetic signal, is related to $Z$ (a quantity of meteorological significance) by $\bar{P}=C Z / r^{2}$ where $C$ is the radar constant and $r$ is the range to the resolution volume. The parameters $a$ and $b$ of the $Z-R$ relation can be estimated by comparing radar measurements of reflectivity $(Z)$ with surface instruments such as raingages (which estimate rain intensity) or raindrop size measuring devices (e.g., disdrometers or optical array probes) which estimate the RSD. These surface devices have extremely small sampling volumes compared to the radar sampling volume. Thus, even under ideal conditions, it is difficult to separate statistical fluctuations from fluctuations caused by physical processes (e.g., the changing RSD) with respect to the relationship between radar $Z$ and surface measured $R$.

In order to overcome the problems associated with acquiring simultaneous radar/surface rainfall data, radar meteorologists often measure the RSD (either at the surface, or in situ, using instrumented aircraft) or approximate the RSD by some functional form, and calculate both reflectivity $\left(Z_{\mathrm{RSD}}\right)$ and rainfall rate from the size spectrum. We use the subscript RSD on $Z$ to denote that it is calculated based on a measured RSD or on an assumed form for the RSD. Hence, it is pos- 
sible to relate $Z_{\mathrm{RSD}}$ and rain intensity, and to translate these relationships to radar measured $Z$ versus rain intensity. If the RSD is approximated by a gamma distribution, the three parameters of the gamma distribution namely, particle number density, shape parameter and scale parameter, are generally unknown but are often estimated using measured RSDs via moment methods or Maximum Likelihood Estimators (MLEs), Ulbrich (1983), Mielke (1976), Wong and Chidambaram (1985). Lognormal RSDs have also been used by Feingold and Levin (1986). Statistical correlations between the various moments of the RSD must be understood before deductions regarding the physical fluctuation of the gamma or lognormal parameters can be made.

Our paper is organized as follows: Section 2 describes the RSD model and intercomparison between radar reflectivity and rainfall intensity. Section 3 considers the theoretical correlation between $Z_{\mathrm{RSD}}$ and rain intensity (as well as other RSD moments) for a gamma RSD. Section 4 considers the statistical fluctuations inherent in the radar measurements of $Z$. Simulation methods for radar $Z$, and gamma RSDs (including moments) are given in section 5. Section 6 describes the results of our simulations which are applied to explain some features of radar/disdrometer rainfall intercomparisons. In section 7 we comment on the $\mathrm{N}_{0^{-}}$ $m$ relationships derived by Ulbrich (1983) and Ulbrich and Atlas (1985).

\section{Raindrop size distribution}

The space-time evolution of the raindrop size distribution (RSD) is typically due to a variety of physical processes, e.g., evaporation, collision-coalescence, collisional breakup, drop sorting, etc. Both cloud models and measurements of RSDs at the surface show that a gamma RSD can account for many of the natural variations in the RSD, Ulbrich (1983):

$$
N(D)=N_{0} D^{m} \exp (-\Lambda D),
$$

where $N(D)$ is the number of raindrops per unit volume per unit size interval $(D$ to $D+\Delta D)$. In terms of the conventional gamma probability density function (pdf), $N(D)$ can be written in the equivalent form,

$$
N(D)=\frac{N_{T}}{\Gamma(\alpha) \beta^{\alpha}} D^{\alpha-1} \exp (-D / \beta),
$$

where $\alpha>0, \beta>0, D \geqslant 0$. We note that $N_{0}=\dot{N}_{T} /$ $\Gamma(\alpha) \beta^{\alpha} ; m=\alpha-1 ; \Lambda=1 / \beta$. A physically meaningful parameter known as the median volume diameter $D_{0}$ can be defined by,

$$
\int_{0}^{D_{0}} D^{3} N(D) d D=\int_{D_{0}}^{\infty} D^{3} N(D) d D
$$

where $D_{0}$ is such that all drops with diameter $\leqslant D_{0}$ contribute to one-half the total liquid water content.
Ulbrich (1983) has shown that $\Lambda D_{0} \approx 3.67+m$. Reflectivity $\left(Z_{\mathrm{RSD}}\right)$ and rain intensity can be formulated as various moments of $N(D)$ :

$$
\begin{aligned}
Z_{\mathrm{RSD}} & =\int_{0}^{\infty} D^{6} N(D) d D \quad\left[\mathrm{~mm}^{6} \mathrm{~m}^{-3}\right] \\
R & =\frac{\pi}{6} \int_{0}^{\infty} D^{3} v(D) N(D) d D \quad\left[\mathrm{~mm} \mathrm{~h}^{-1}\right]
\end{aligned}
$$

where $v(D)=C D^{0.67}$, is the raindrop still air fall speed, Atlas and Ulbrich (1977). Note that $C=17.67 \mathrm{~m}$ $\mathrm{s}^{-1} \mathrm{~cm}^{-0.67}$.

RSDs can be estimated by using surface instruments, e.g., disdrometers (drop size meters), or using probes mounted on instrumented aircraft. The Joss-Waldvogel (1967) disdrometer is a momentum device with a sensor area of $50 \mathrm{~cm}^{2}$, and estimates $N(D)$ for $D$ in the range $0.5-5 \mathrm{~mm}$ with typical integration times of $30 \mathrm{sec}-1 \mathrm{~min}$. Even though the sampling volume changes with drop diameter, a typical volume of 0.9 $\mathrm{m}^{3}$ corresponds to $2 \mathrm{~mm}$ drop size and $30 \mathrm{sec}$ averaging. The 2D-PMS precipitation probe has a sampling volume of $0.1 \mathrm{~m}^{3} \mathrm{~s}^{-1}$ for an aircraft speed of $100 \mathrm{~m} \mathrm{~s}^{-1}$. A number of authors have used $N(D)$ data from JossWaldvogel disdrometers, Ulbrich (1983), Joss and Gori (1978), Bringi et al. (1982), Goddard et al. (1982), Seliga et al. (1986). In the Rayleigh scattering limit the radar reflectivity factor $Z$ is defined as

$$
Z=\frac{1}{\Delta V} \sum_{i} D_{i}^{6} \quad\left[\mathrm{~mm}^{6} \mathrm{~mm}^{-3}\right]
$$

where the summation applies to raindrops within a volume $\Delta V$. Since the range of $Z$ can be quite large we define $\mathrm{dBZ}=10 \log _{10}(Z)$.

There is a general correlation between $Z$ and rain intensity expressed by a power law of the form $Z=a R^{b}$. Zawadzki (1984) has used the data of Richards and Crozier (1983) to intercompare radar $Z$ measurements with surface disdrometer measurements of rain intensity. He has analyzed a number of factors which can cause discrepancies between radar-derived rain rates and surface-measured rain rates. Sampling errors affect both the surface measurements (due to inadequate sample volume) as well as the radar measurements of mean power (or $\mathrm{dBZ}$ ) due to the finite Doppler velocity spectrum. Systematic errors can be caused by radar calibration problems, as well as by the noncoincidence (in space-time) of the raindrops measured by the radar as opposed to the raindrops which actually impact on the surface disdrometer. Figure la taken from Richards and Crozier (1983) shows a scatter plot of rain intensity versus $Z_{\mathrm{RSD}}$ [estimated from a Joss-Waldvogel disdrometer using Eq. (6)], while Fig. 1b shows rain intensity versus radar-measured $Z$. Zawadzki (1984) notes that the variability in $R$ for a given $Z_{\mathrm{RSD}}$ is significantly less ( $\sim$ factor of 3 ) than the variability in $R$ for the same radar-measured $Z$. We have simulated a similar ex- 
a

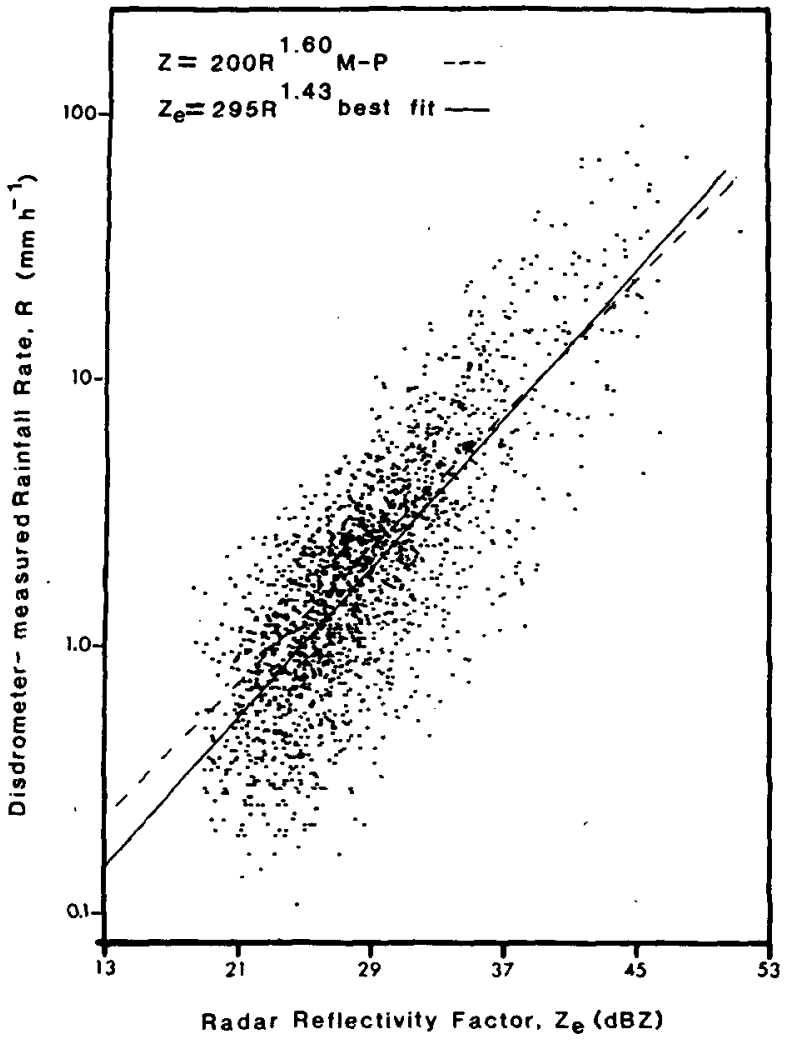

C

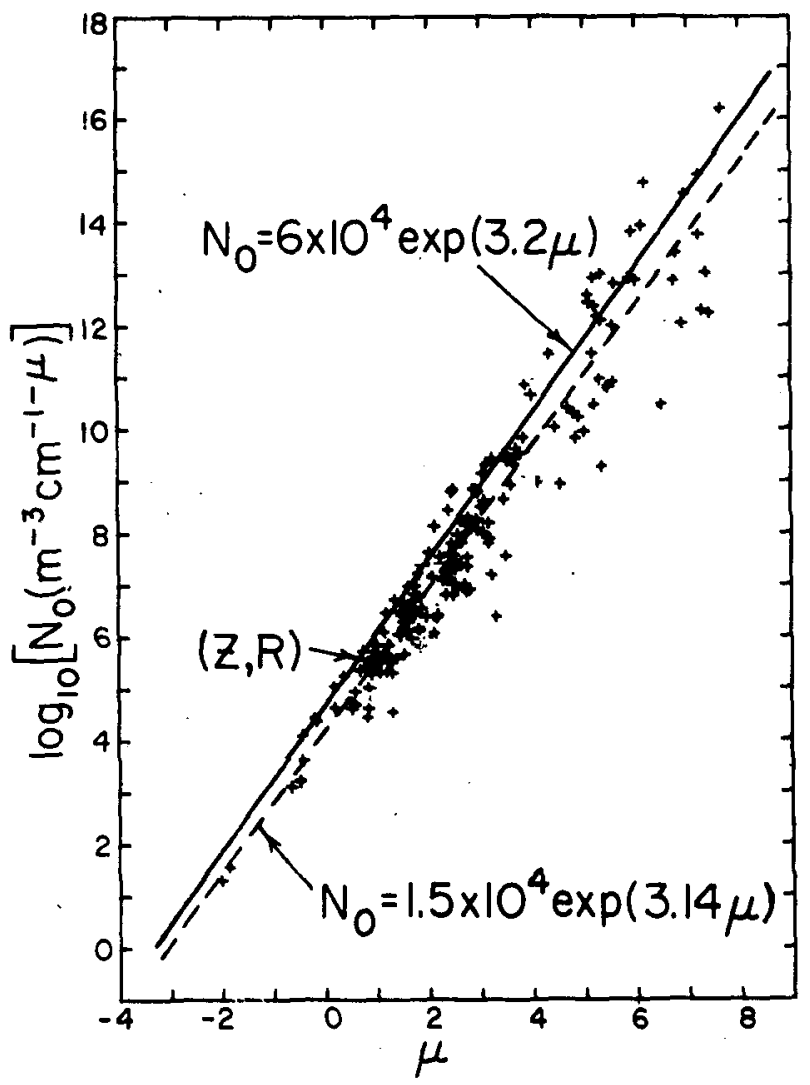

b

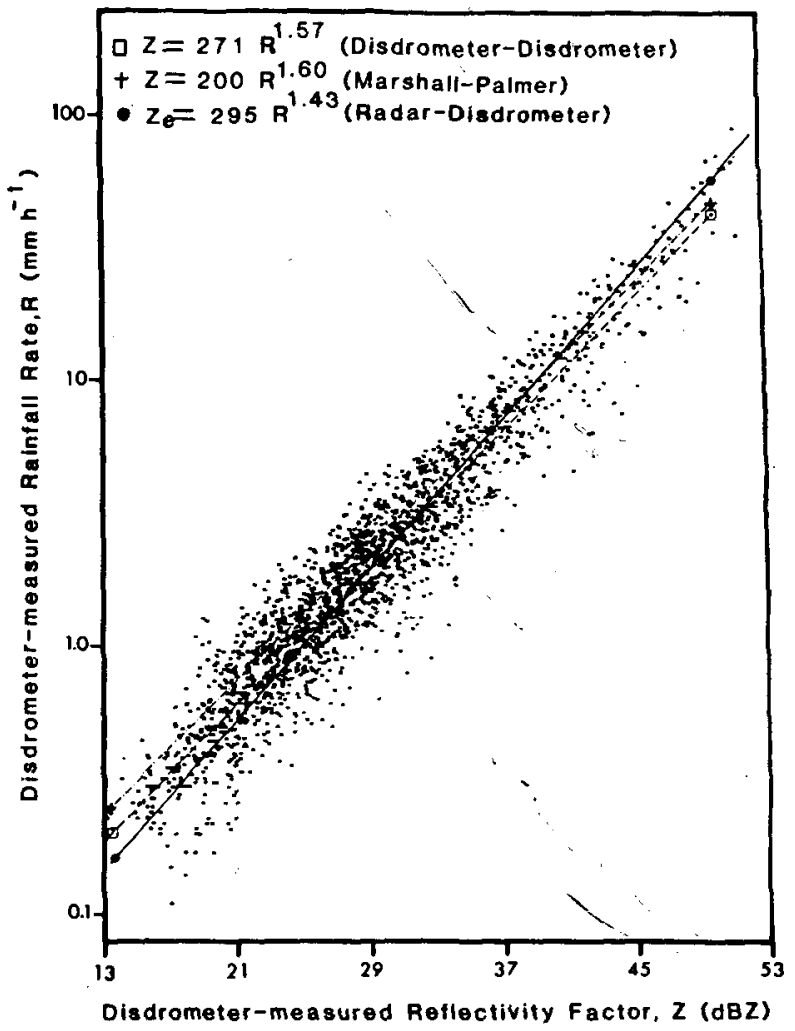

FIG. 1a. Scattergram of disdrometer derived values of $Z$ and $R$ for data in the Toronto, Ontario, region taken during 1977-79 summer seasons (from Richards and Crozier, 1983). Each point represents a 7 min sample. (Reproduced by permission from Atmosphere-Ocean.) FIG. 1b. Scattergram of radar measured $Z$ and disdrometer derived $R$ for the same events as in Fig. Ia (from Richards and Crozier). (Reproduced by permission from Atmosphere-Ocean.)

Fig. 1c. Plot of the gamma RSD parameters $N_{0}$ versus $m$ as deduced from experimental dropsize spectra from moment to moment within a given rainfall type. The dashed line is the least squares fit to all the data. The solid line is the least squares $N_{0}-m$ line from empirical $Z$ $R$ relationships. Also shown as $(Z, R)$ is the point deduced from empirical $Z-R$ relation which applies to these data (from Ulbrich, 1983). Note $m$ is the same as $\mu$. 
periment to study the features of the variability, noted by Zawadzki, by concentrating solely on experimental factors.

As another example, we consider the relationship between $N_{0}$ and $m$ [see Eq. (1)] derived by Ulbrich (1983) using RSDs measured by a Joss-Waldvogel disdrometer. Ulbrich estimates $N_{0}, m$ and $D_{0}$ based on higher moments of the RSD. This procedure places more weight on the larger drop sizes as compared to MLEs which place more emphasis on drop sizes having a higher frequency of occurrence (Wong and Chidambaram, 1985). Figure 1c shows a scatter plot of $\log _{10} N_{0}$ versus $m$ taken from Ulbrich (1983). From this data, Ulbrich concludes that physical processes result in a $N_{0}-m$ relation, and that, effectively, the three-parameter gamma RSD reduces to a two-parameter form. We have simulated this experiment and show in section 5 that the relationship between $N_{0}$ and $m$ could be due to the statistical correlations between estimators of the higher order moments of the RSDs. The above two examples imply that statistical fluctuations must be separated from variations induced by physical causes. Simulations offer a powerful method of studying the statistics of radar and surface measurements where the "natural" fluctuations can be introduced separately. However, such simulations involve large-scale computations on a supercomputer since the physical parameters must be varied over a wide range.

\section{Surface disdrometer measurements}

Gertzman and Atlas (1977) have shown that, in raindrop sampling devices such as disdrometers, the measurement variability is due both to statistical sampling errors and to real fine-scale physical variations not readily separable from the statistical ones. Sasyo (1965) and Cornford (1967) have shown that, for a constant mean rain intensity, the total number of raindrops observed will be distributed about its mean according to the Poisson distribution. This property has been used by Joss and Waldvogel (1969), Gertzman and Atlas (1977) and Wong and Chidambaram (1985) to obtain the fractional standard deviations of higherorder moment estimators (which correspond to radar measurements) of RSDs. In this work we use a somewhat different approach so that the correlation between higher-order RSD moment estimators can be computed.

We rewrite Eq. (2) in the form of a gamma pdf,

$$
f(D)=\frac{\Lambda^{m+1}}{\Gamma(m+1)} D^{m} \exp (-\Lambda D) \text {. }
$$

In the following development we assume that the sampling volume, $V$, is constant and does not vary with raindrop size. If $V$ does vary with $D$, then this dependency can be introduced by multiplying $f(D)$ by the sampling volume function $V(D)$, see appendix $C$.

If $n$ raindrops are observed within a fixed sample volume $V$ with diameters $D_{1}, D_{2} \ldots D_{\mathrm{n}}$, then this RSD is a composite distribution of total number of raindrops (or equivalently, the concentration of drops within any interval $D$ to $D+\Delta D$ ) and the drop diameter, where the diameters are distributed according to the gamma pdf, and the total number of raindrops (n) are distributed according to the Poisson distribution.

Conventional estimators of higher-order RSD moments are expressed as follows:

$$
\begin{aligned}
Z & =\frac{1}{V} \sum_{i=1}^{\mathrm{n}} D_{i}^{6}, \quad\left[\mathrm{~mm}^{6} \mathrm{~m}^{-3}\right] \\
R & =\frac{7.12 \times 10^{-3}}{V} \sum_{i=1}^{\mathrm{n}}{D_{i}}^{3.67}, \quad\left[\mathrm{~mm} \mathrm{~h}^{-1}\right] \\
\mathrm{LWC} & =\frac{\pi}{6 V} \sum_{i=1}^{\mathrm{n}} D_{i}^{3}, \quad\left[\mathrm{gm} \mathrm{m}^{-3}\right]
\end{aligned}
$$

where LWC stands for liquid water content. The above estimators can be written in general as

$$
\hat{p}_{\alpha}=\frac{C_{\alpha}}{V} \sum_{i=1}^{\mathbf{n}} D_{i}^{\alpha}
$$

where $p_{\alpha}=C_{\alpha} \int D^{\alpha} N(D) d D$. We can now find the mean of $\hat{p}_{\alpha}$ as

$$
\mathrm{E}\left(\hat{p}_{\alpha}\right)=\frac{C_{\alpha}}{V} \mathrm{E}\left(\sum_{i=1}^{\mathbf{n}} D_{i}^{\alpha}\right)
$$

where E( ) stands for the expected value. The above expectation is that of a random sum, hence

$$
\begin{aligned}
\mathrm{E}\left(\sum_{i=1}^{\mathbf{n}} D_{i}^{\alpha}\right) & =\mathrm{E}\left(\mathrm{E}\left\{\sum_{i=1}^{\mathbf{n}} D_{i}^{\alpha} \mid \mathbf{n}\right\}\right) \\
& =\mathrm{E}_{A}(\mathbf{n}) \mathrm{E}_{B}\left(D^{\alpha}\right),
\end{aligned}
$$

where $\mathrm{E}_{A}(\quad)$ is the expectation with respect to the total number of raindrops and $E_{B}(\quad)$ is the expectation with respect to the gamma pdf. $\mathrm{E}\left(-\left.\right|_{-}\right)$refers to conditional expectation. Hence,

$$
\begin{aligned}
\mathrm{E}_{A}(\mathbf{n}) & =V N_{T}=V N_{0} \frac{\Gamma(m+1)}{\Lambda^{m+1}} \\
\mathrm{E}_{B}\left(D^{\alpha}\right) & =\frac{\Gamma(m+\alpha+1)}{\Gamma(m+1) \Lambda^{\alpha}} \\
\mathrm{E}\left(\hat{p}_{\alpha}\right) & =C_{\alpha} N_{T} \frac{\Gamma(m+\alpha+1)}{\Gamma(m+1) \Lambda^{\alpha}} .
\end{aligned}
$$

It is easily verified that $p_{\alpha}$ is an unbiased estimator of $p_{\alpha}$. Similarly,

$$
\begin{aligned}
\operatorname{var}\left(\hat{p}_{\alpha}\right) & =\frac{C_{\alpha}{ }^{2}}{V^{2}} \operatorname{var}\left(\sum_{i=1}^{\mathrm{n}} D_{i}^{\alpha}\right) \\
& =\frac{C_{\alpha}{ }^{2} N_{0}}{V} \frac{\Gamma(m+2 \alpha+1)}{\Lambda^{m+2 \alpha+1}} .
\end{aligned}
$$


We note that the variance decreases with increase in sample volume, as expected. The fractional standard deviation (FSD) of $\hat{p}_{\alpha}$ is

$$
\operatorname{FSD}\left(\hat{p}_{\alpha}\right)=\left[\frac{\Lambda^{m+1}}{N_{0} V}\right]^{1 / 2} \frac{\Gamma^{1 / 2}(m+2 \alpha+1)}{\Gamma(m+\alpha+1)} .
$$

Equation (12) is identical to Eq. (29) of Gertzman and Atlas (1977).

We now derive the correlation between two estimators $\hat{p}_{\alpha}$ and $\hat{p}_{\beta}$, defined as

$$
\begin{aligned}
& \hat{p}_{\alpha}=\frac{C_{\alpha}}{V} \sum_{i=1}^{\mathbf{n}} D_{i}^{\alpha} \\
& \hat{p}_{\beta}=\frac{C_{\beta}}{V} \sum_{i=1}^{\mathbf{n}} D_{i}^{\beta} .
\end{aligned}
$$

The covariance between $\hat{p}_{\alpha}$ and $\hat{p}_{\beta}$ is

$$
\operatorname{cov}\left(\hat{p}_{\alpha}, \hat{p}_{\beta}\right)=\frac{C_{\alpha} C_{\beta}}{V^{2}} \operatorname{cov}\left(\sum_{i=1}^{\mathrm{n}} D_{i}^{\alpha}, \sum_{i=1}^{\mathrm{n}} D_{i}^{\beta}\right) .
$$

By conditioning on $\mathbf{n}$ the covariance can be written as

$$
\begin{aligned}
\operatorname{cov}\left(\sum_{i=1}^{\mathbf{n}} D_{i}^{\alpha}, \sum_{i=1}^{\mathbf{n}} D_{i}^{\beta}\right) & =\mathrm{E}\left\{\operatorname{cov}\left(\sum_{i=1}^{\mathbf{n}} D_{i}^{\alpha}, \sum_{i=1}^{\mathbf{n}} D_{i}^{\beta}\right) \mid \mathbf{n}\right\} \\
& +\operatorname{cov}\left\{\mathrm{E}\left(\sum_{i=1}^{\mathbf{n}} D_{i}^{\alpha} \mid \mathbf{n}\right), \mathrm{E}\left(\sum_{i=1}^{\mathbf{n}} D_{i}^{\beta} \mid \mathbf{n}\right)\right\} .
\end{aligned}
$$

Since the $D_{i}$ are independent and identically distributed (iid), $\operatorname{cov}\left[D_{i}^{\alpha}, D_{j}^{\beta}\right]=0$ when $i \neq j$. Therefore, the first term on the right-hand side of Eq. (14) simplifies to $\mathrm{E}(\mathrm{n}) \operatorname{cov}\left(D^{\alpha}, D^{\beta}\right)$, while the second term simplifies to $\mathrm{E}\left(D^{\alpha}\right) \mathrm{E}\left(D^{\beta}\right) \operatorname{var}(\mathbf{n})$. Thus,

$$
\begin{aligned}
\operatorname{cov}\left(\hat{p}_{\alpha}, \hat{p}_{\beta}\right) & =\frac{C_{\alpha} C_{\beta}}{V^{2}} \mathrm{E}(\mathbf{n}) \mathrm{E}\left(D^{\alpha+\beta}\right) \\
& =\frac{C_{\alpha} C_{\beta} N_{0}}{V} \frac{\Gamma(m+\alpha+\beta+1)}{\Lambda^{m+\alpha+\beta+1}} .
\end{aligned}
$$

Combining with Eq. (11b) we get the correlation coefficient $\rho_{\alpha, \beta}$ as

$$
\rho_{\alpha, \beta}=\frac{\Gamma(m+\alpha+\beta+1)}{\{\Gamma(m+2 \alpha+1) \Gamma(m+2 \beta+1)\}^{1 / 2}} .
$$

The above equations are valid for the standard gamma distribution with integration limits of 0 and $\infty$ for the diameter $D$. It is interesting to note that the correlation coefficient between $\hat{p}_{\alpha}$ and $\hat{p}_{\beta}$ is independent of $N_{0}$ and $D_{0}$. In Fig. 2 we show plots of $\rho_{\alpha, \beta}$ versus $m$, where $\hat{p}_{6}$ $=Z_{\mathrm{RSD}}$ (radar reflectivity) while $\hat{p}_{\beta}$ represents estimators with $\beta=0$ (concentration), $\beta=1$ (mean raindrop size), $\beta=2$ (optical extinction), $\beta=3$ (liquid water content), and $\beta=3.67$ (rain intensity, $R$ ). Note that estimators of $Z_{\mathrm{RSD}}$ and $R$ are highly correlated and nearly independent of the shape $(m)$ of the gamma pdf.

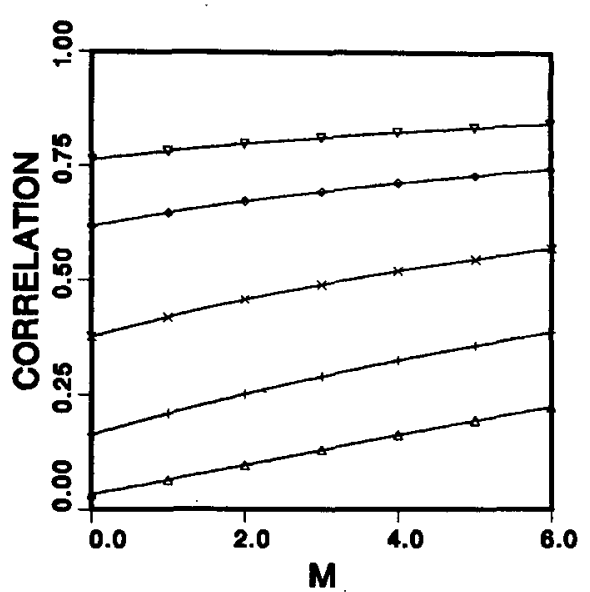

FiG. 2. Correlation of reflectivity, $\mathrm{dBZ}$, with other meteorological quantities of interest plotted as a function of $m$. The various curves represent correlation of $\mathrm{dBZ}$ with $(\Delta)$ concentration, $(+)$ mean particle size, $(\times)$ mean particle size squared, $(\diamond)$ liquid water content and $(\nabla)$ rainfall rate. Note that the correlation is due to statistical fluctuations only.

Hence, experimental data which show scatter plots of $Z_{\mathrm{RSD}}$ versus $R$ obtained from disdrometers must be carefully interpreted, i.e., experimentally derived correlations will contain the effects of both physical correlations as well as statistical correlations.

In the above analysis we have derived the correlation between the estimates assuming the sample volume is filled with drops that come from one RSD. In appendix A we show that the correlation between the estimates is still high even if we had the RSD parameters varying in the sample volume, and the expression for correlation is the same as Eq. (16) for mixed RSDs under the condition that the $m$ parameter is the same.

In order to simulate $\hat{p}_{\alpha}$ we need to derive its distribution function. Since $\hat{p}_{\alpha}$ is a moment estimator with a random sum, analytic derivations are hopelessly complicated, especially for values of $\alpha>0$. Hence, we resort to simulating $\hat{p}_{\alpha}$ (for $\alpha=6$ and 3.67) by first simulating the RSD. Typical concentrations of raindrops vary from $10^{2}$ to $10^{5} \mathrm{~m}^{-3}$ (Gordon and Marwitz, 1984). Considering a $100 \mathrm{~L}\left(0.1 \mathrm{~m}^{3}\right)$ sample volume, the number of raindrops can vary between 10 to 10000 and the number increases with sample volume. To simulate the variability in concentration we have to use Poisson deviates. Then for each of these numbers (n) we need to simulate $n$ gamma deviates to represent the RSD. Next the physical parameters $\left(N_{0}, m\right.$ and $\left.D_{0}\right)$ of the gamma RSD must be varied over the range of observed values. For example, consider $N_{0}=8000$ $\mathrm{mm}^{-1} \mathrm{~m}^{-3}, D_{0}=2 \mathrm{~mm}$ and $m=0$ (exponential RSD) and $V=0.1 \mathrm{~m}^{3}$. To obtain a scatter plot of $Z_{\mathrm{RSD}}$ versus $R$ under these conditions it takes 125 seconds of execution time on the CSU/Cyber $180 / 830$ computer. It is apparent that large computing power is needed to handle such intensive computational needs. 


\section{Radar measurements}

Radar measurements of reflectivity $Z$ involve estimation of $Z$ from the measurement of mean (time averaged) backscattered power $(\bar{P})$ from a given radar resolution volume, Doviak and Zrnić (1984). To obtain the mean power, $\bar{P}$, a large number of power samples must be averaged. The total number of samples depends on the radar pulse repetition time (PRT), and the dwell time of the antenna beam on the resolution volume. Doviak and Zrnić (1984) show that the power samples have exponential marginal distributions. Zrnić (1975) has developed a procedure for simulating the time series of power samples assuming a Gaussian Doppler velocity spectrum. We use his method in our work and refer to Zrnić's paper for details. The principal assumptions we made are as follows (for a typical meteorological radar):

Doppler Spectrum

Radar Reflectivity
Pulse Repetition Time
Number of Samples
Radar Wavelength
Receiver

Our radar simulations, for a given mean power (or, reflectivity) involve length $N(128)$ exponential deviates, and length $N$ complex FFTs. As the reflectivity is varied (by changing $N_{0}, D_{0}$, or $m$ ) we see that the radar simulations are also computationally intensive.

\section{Simulations}

As discussed in sections 2 and 3, the surface disdrometer and radar simulations are computationally intensive, requiring simulations of gamma, Poisson and exponential deviates, as well as complex FFTs. These simulations are repeated many times as the physical parameters, namely, $N_{0}, D_{0}$ and $m$ of the RSD are varied over a considerable range of values commonly found in rainfall.

\section{a. Exponential random deviates}

The inverse $\mathrm{CDF}$ technique for generating exponentials is used here. We generate them from uniform $(0$, 1) deviates and take the negative logarithm. This method is easily vectorizable since the CDF is in closed form and conditional checks for specific values can be avoided. Exponentials with means differing from unity are needed here. Some timing runs made on the CSU/ Cyber 205 indicate that a length 100 string of deviates can be computed 2.2 times faster than the scalar method, whereas the speed-up factor increases to 4.8 and 6.4 for strings of length 500 and 2500 , respectively. This speed-up factor is important in our work since a large number of these simulations are needed.

\section{b. Gamma random deviates}

Kennedy and Gentle (1980) discuss a number of methods of simulating gamma random deviates. Among the various algorithms, the one proposed by Cheng (1978) appears to be most suitable for vector implementation. This method is an adaptation of the envelope rejection technique and is described in appendix $B$.

\section{Discussion and results}

We now apply our simulation results to two types of problems, namely

(i) radar-surface disdrometer intercomparisons, and

(ii) inferences on gamma RSD parameters using surface disdrometer measurements.

\section{a. Radar/disdrometer intercomparisons}

In Figs. 3-5 we show our simulation results where Figs. 3a-5a show scatter plots of surface rain intensity versus $Z_{\mathrm{RSD}}$, while Figs. $3 \mathrm{~b}-5 \mathrm{~b}$ show scatter plots of rain intensity versus radar $Z$. We have used a fixed sample volume of $0.1 \mathrm{~m}^{3}$ to illustrate the statistical features. The physical parameters $N_{0}, D_{0}$ and $m$ are varied differently in each Fig. 3-5. For example, in Fig. $3 \mathrm{a}$ and $3 \mathrm{~b}$ we represent one rainfall condition with $N_{0}$ $=8000 \mathrm{~mm}^{-1} \mathrm{~m}^{-3}, D_{0}=2 \mathrm{~mm}$ and $m=0$. Figure $3 \mathrm{a}$ shows very good correlation between $R$ and $Z_{\mathrm{RSD}}$ whereas in Fig. 3b, $R$ appears uncorrelated with radar $Z$. In Fig. 3b note that the distribution of $R$ is asymmetric. We now vary the physical parameter $m$ from 0.5 to 5 in Figs. $4 \mathrm{a}$ and $4 \mathrm{~b}$ with the same $N_{0}, D_{0}$ values as before. The correlation in Fig. $4 \mathrm{a}$ is significantly higher than in Fig. 4b. In Figs. $5 \mathrm{a}$ and $5 \mathrm{~b}$ we keep $N_{0}$ $=8000 \mathrm{~mm}^{-1} \mathrm{~m}^{-3}, m=0$ and vary $D_{0}$ from 0.8 to 2.8 $\mathrm{mm}$. In Fig. $6 \mathrm{a}$ and $6 \mathrm{~b}$ we vary $N_{0}, D_{0}$ and $m$ simultaneously over a broad range of values that can physically occur, but the sample volume is increased to 0.3 $\mathrm{m}^{3}$. Again, the same feature is deduced, i.e., $R$ versus $Z_{\mathrm{RSD}}$ is more tightly correlated than $R$ versus radar $Z$ over a wide variety of physical rainfall conditions. In Figs. $6 \mathrm{c}$ and $6 \mathrm{~d}$ the simulations are repeated with sample volume increased to $0.9 \mathrm{~m}^{3}$, which corresponds to $1 \mathrm{~km}$ path average for a 2D-PMS precipitation probe. We note the scatter in Fig. $6 \mathrm{~d}$ is considerably reduced from Fig. 6b, as expected, but still more than the scatter in Fig. 6c. It is obvious that in the limit of infinite sample volume figure pairs such as Figs. $6 \mathrm{a}$ and $6 \mathrm{~b}$ will show identical scatter which would represent the physical relationship between $R$ and $Z$. Our results are obtained for an ideal, noise-free radar with the radar resolution volume completely filled by a homogeneous rain medium identical to that sampled by the disdrometer. In appendix $\mathrm{C}$ we consider the effects of sample volume varying with size.

In practical applications the sample volume is often 

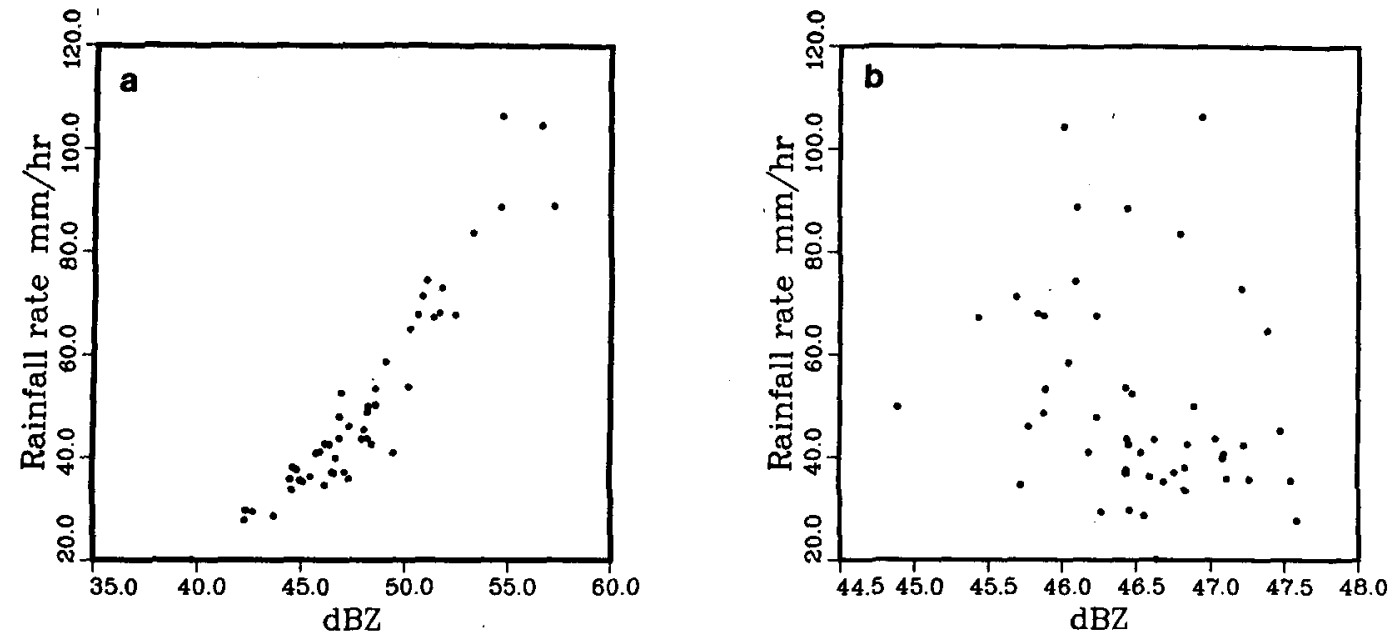

FIG. 3. (a) Scatter plot of rainfall rate versus $\mathrm{dBZ}$ where both are derived from the drop spectra observed by disdrometer. This figure shows 50 realizations under the condition $N_{0}=8000 \mathrm{~mm}^{-1} \mathrm{~m}^{-3}, D_{0}=2 \mathrm{~mm}, m=0$. Sample volume is $0.1 \mathrm{~m}^{3}$. (b) As in (a) except dBZ is "measured" by radar. Note that the scale of Fig. 3b is not the same as Fig. 3a and is enhanced in scale to see the features clearly.

increased by longer time averaging, e.g., the 7-min averaging time used by Richards and Crozier (1983), or longer path averages $(5-10 \mathrm{~km})$ in the case of 2D-PMS probes. In convective rainfall the space-time evolution of RSDs can often be quite rapid, see for example the fast response ( $1 \mathrm{sec}$ ) raingage data of Semplak and Turrin (1969). In addition, advection of rain cells over the disdrometer introduces changes in the mean observed RSD. To overcome these problems Goddard and Cherry (1984) used $30 \mathrm{sec}$ disdrometer data in their radar/disdrometer comparisons. In the simulations shown in Fig. 6a-d we reemphasize that both radar and disdrometer sample identical, homogeneous RSDs, and this assumption breaks down when long time or path averages are used, as in the case of disdrometer or 2D-PMS probes, respectively. In appendix A we consider a simple model simulation to account for mixing RSDs with different mean properties. In this model the fractional contributions of the individual RSDs in the mixture are different for disdrometer (i.e., linear) as compared to radar (i.e., areal). The simulation results show that radar/disdrometer scatter can vary considerably as compared to the disdrometer-based data alone. In order to avoid long time averaging of the disdrometer data it is preferable to use several disdrometers sampling the same mean RSD, especially in
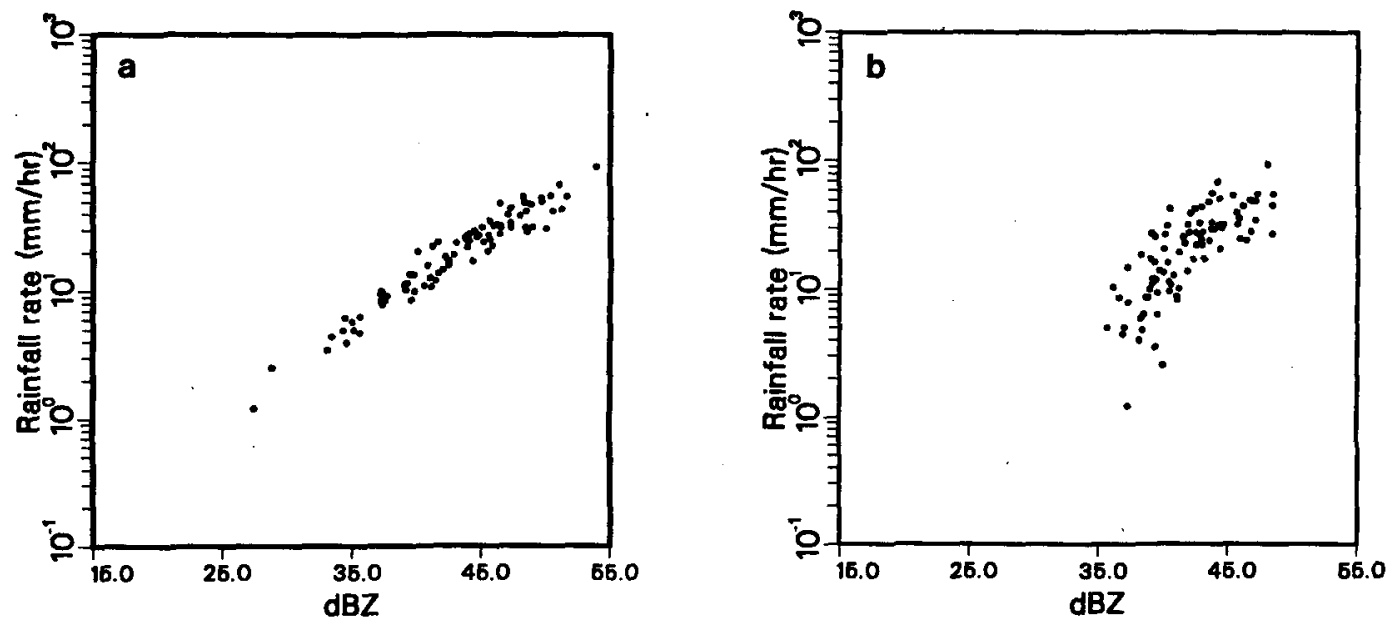

FIG. 4. (a) Scatter plot of rainfall rate versus $\mathrm{dBZ}$ where both are derived from drop size spectra. This figure corresponds to $N_{0}=8000 \mathrm{~mm}^{-1} \mathrm{~m}^{-3}, D_{0}=2.0 \mathrm{~mm}$ and $m$ varying between 0.5 to 5 . Sample volume is $0.1 \mathrm{~m}^{3}$. (b) As in (a) except $\mathrm{dBZ}$ is "measured" by radar. 

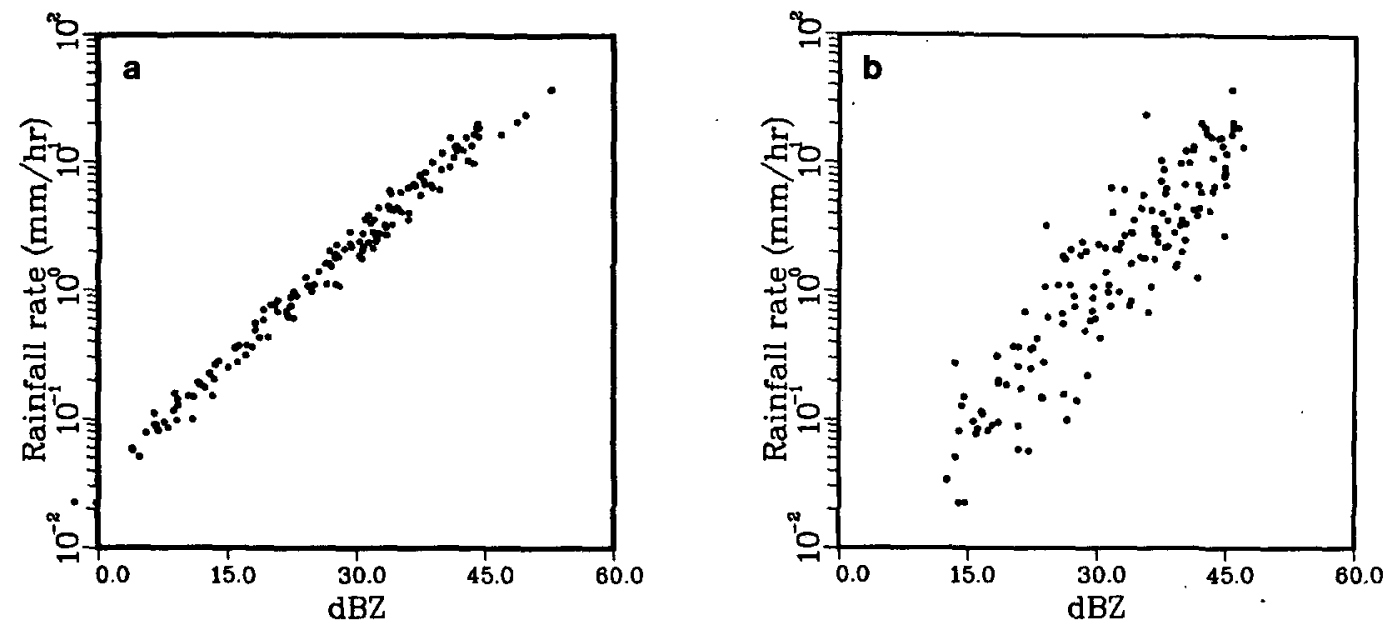

FIG. 5. As in Fig. 4a, b but with $N_{0}=8000 \mathrm{~mm}^{-1} \mathrm{~m}^{-3}, m=0$ and $D_{0}$ varying between 0.8 and $2.8 \mathrm{~mm}$. Sample volume is $0.1 \mathrm{~m}^{3}$.
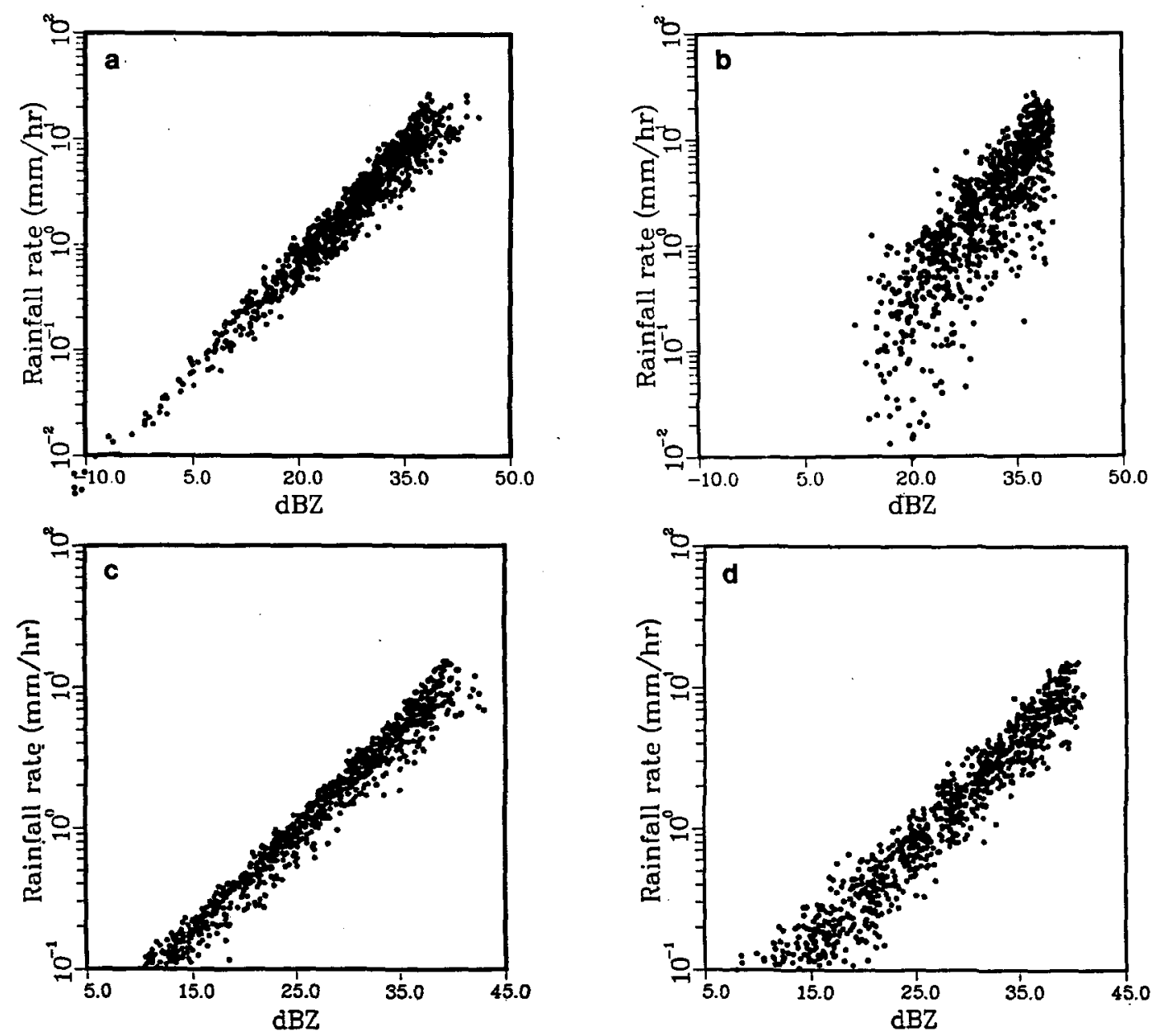

Fig. 6. (a) Comprehensive scatter plot of rainfall rate versus $\mathrm{dBZ}$, both of which are derived from drop size spectra. Variation of $N_{0}, m$ and $D_{0}$ cover a wide range of suggested $Z-R$ relationships, Ulbrich (1983). Sample volume is 0.3 $\mathrm{m}^{3}$. (b) As in (a) but with $\mathrm{dB} Z$ "measured" by radar. The Doppler spectrum width has been varied linearly between 1 and $6 \mathrm{~m} \mathrm{~s}^{-1}$ in proportion to reflectivity. (c) As in (a) but for sample volume $0.9 \mathrm{~m}^{3}$. (d) As in (c) but with dBZ "measured" by radar. The Doppler spectrum width has been varied linearly between 1 and $6 \mathrm{~m} \mathrm{~s}^{-1}$ in proportion to reflectivity. 
convective rainfall. While the physical factors enumerated by Zawadzki (1984) are important for comparing radar rainfall aloft with surface disdrometer measurements, our simulation results show that, even for ideal conditions, the decorrelation between radar reflectivity and disdrometer rainfall must be taken into account since these are different stochastic processes.

\section{b. Disdrometer inferences}

In section 1 we discussed Ulbrich's (1983) conclusion that the three-parameter gamma RSD in fact reduces to a two-parameter gamma RSD with $N_{0}$ and $m$ being related by $N_{0}=6 \times 10^{4} \exp (3.2 \mathrm{~m})$, see Fig. $1 \mathrm{c}$. Ulbrich (1983) estimates the parameters $N_{0}, D_{0}$ and $m$ from disdrometer measured RSDs using three moment estimators, viz.,

$$
\begin{gathered}
G^{\prime}=\frac{\int_{0}^{\infty} D^{6} N(D) d D}{D_{m}^{3} \int_{0}^{\infty} D^{3} N(D) d D}=\frac{(6+m)(5+m)}{(4+m)^{2}} \\
D_{m}=\frac{\int_{0}^{\infty} D^{4} N(D) d D}{\int_{0}^{\infty} D^{3} N(D) d D}=\left(\frac{4+m}{3.67+m}\right) D_{0} \\
\mathrm{LWC}=\frac{\pi}{6} \int_{0}^{\infty} D^{3} N(D) d D \\
=0.52 \Gamma(4+m) N_{0}\left[\frac{D_{0}}{3.67+m}\right]^{m+4} .
\end{gathered}
$$

Using experimentally measured RSDs, $G^{\prime}, D_{m}$ and LWC are calculated using discrete versions of the integral in Eqs. (17a, b, c), from which the gamma RSD parameters $N_{0}, D_{0}$ and $m$ are inferred. The result of this "inversion" procedure is given as a scatter plot of $\log _{10} N_{0}$ versus $m$ in Fig. 1c which is taken from Ulbrich (1983). If a linear relationship between $\log _{10} N_{0}$ and $m$ exists for natural gamma-parameterized RSDs, then in essence the gamma RSD is reduced to a two-parameter form.

To study the statistical fluctuations in the moment estimators defined by Eqs. (17a, b, c) we use the Ulbrich inversion procedure with simulated gamma RSDs. In Figs. 7a-c we assume gamma RSDs with $N_{0}=8000$ $\mathrm{mm}^{-1} \mathrm{~m}^{-3}, m=0$ and $V=1 \mathrm{~m}^{3}$, while $D_{0}$ is $2 \mathrm{~mm}$, $1 \mathrm{~mm}$ and $0.5 \mathrm{~mm}$, respectively. Observe in these figures that estimates of $\log _{10} N_{0}$ vary linearly with estimates of $m$, and the slopes are within the range theoretically derived by Ulbrich (1983). Estimates of $m$ less than -1 are observed in Figs. 7a-c although the distributions had only $m=0$. Hence if we observe estimates of $m$ less than -1 , it does not imply that we have to include those values in the RSD model since the standard gamma distribution is not defined for $m$
$<-1$. If we need to model $m<-1$ in the RSD we have to qualify it with a minimum drop size, $D_{\min }>0$.

In Fig. 8a, $N_{0}, D_{0}$ and $m$ are varied (without any correlation) over a wide range to encompass the full range of naturally occurring RSDs. The scatter points lie in the region derived theoretically by Ulbrich (1983) using a large number of empirical $Z=a R^{b}$ relations. Comparison of Fig. $8 \mathrm{~b}$ with Fig. $1 \mathrm{c}$ also shows that our simulated $\left(N_{0}, m\right)$ pairs lie in the region of experimental scatter derived by Ulbrich (1983). This raises the obvious question of whether the $N_{0}-m$ relationship derived from disdrometer measurements by Ulbrich is due to physical causes or whether it is due to statistical correlations between the various moment estimators defined in Eq. (17). Finally, in Figs. 9 and 10 we show scatter plots of $m$ versus $D_{0}$, and $N_{0}$ versus $D_{0}$ using the simulations. Since these scatter plots indicate the correlation between the estimates is quite low it implies that if significant correlations between these parameters are observed, then it is a real physical observation.

\section{Note on the $N_{0}-m$ relationship}

In the previous section we have shown that the various moments $\left(G^{\prime}, D_{m}\right.$ and LWC) used to arrive at a mean $N_{0}-m$ relation are statistically correlated. It would be desirable to estimate $G^{\prime}, D_{m}$ and LWC from three independent disdrometers placed in proximity and sampling the same rainfall, rather than estimating them from a single disdrometer. If three disdrometers are used the estimates $G^{\prime}, D_{m}$ and LWC would be statistically uncorrelated, and the resulting $N_{0}-m$ relation (if it at all existed) could be ascribed to physical causes. However, the discussion in section $6 \mathrm{~b}$ cannot be used to negate the existence of $N_{0}-m$ relations; it can only show that the particular moment method using a single disdrometer leads to an "artificial" $N_{0}-m$ relation of the form $N_{0}=C e^{k m}$. In this section we explore the functional nature of $N_{0}-m$ relations derived by Ulbrich and Atlas (1985) and Ulbrich (1983) using $Z-R$ and optical extinction- $R$ relationships.

Integral quantities like $Z$ or $R$ can be represented in general form as,

$$
p_{\alpha}=C_{\alpha} \cdot \frac{\Gamma(m+\alpha+1)}{(3.67+m)^{m+\alpha+1}} N_{0} \cdot D_{0}^{m+\alpha+1}
$$

where $p_{\alpha}$ is the $D^{\alpha}$ th moment of a gamma RSD.

If $Z=a R^{b}$, then Ulbrich (1983) has shown that the $N_{0}-m$ relationship depends on $a$. We consider two $N_{0^{-}}$ $m$ relationships which correspond to two values of $a$; the $a$-values chosen span the range normally found in rainfall. These two relations can be represented as $N_{0,1}$ $=C_{1} \exp \left(k_{1} m\right)$ and $N_{0,2}=C_{2} \exp \left(k_{2} m\right)$. For given values of $D_{0}$ and $m$, let $p_{\alpha, 1}$ and $p_{\alpha, 2}$ be defined as

$$
\begin{aligned}
& p_{\alpha, 1}=\frac{\Gamma(\alpha+m+1)}{(3.67+m)^{\alpha+m+1}} N_{0,1} D_{0}^{\alpha+m+1} \\
& p_{\alpha, 2}=\frac{\Gamma(\alpha+m+1)}{(3.67+m)^{\alpha+m+1}} N_{0,2} D_{0}^{\alpha+m+1} .
\end{aligned}
$$



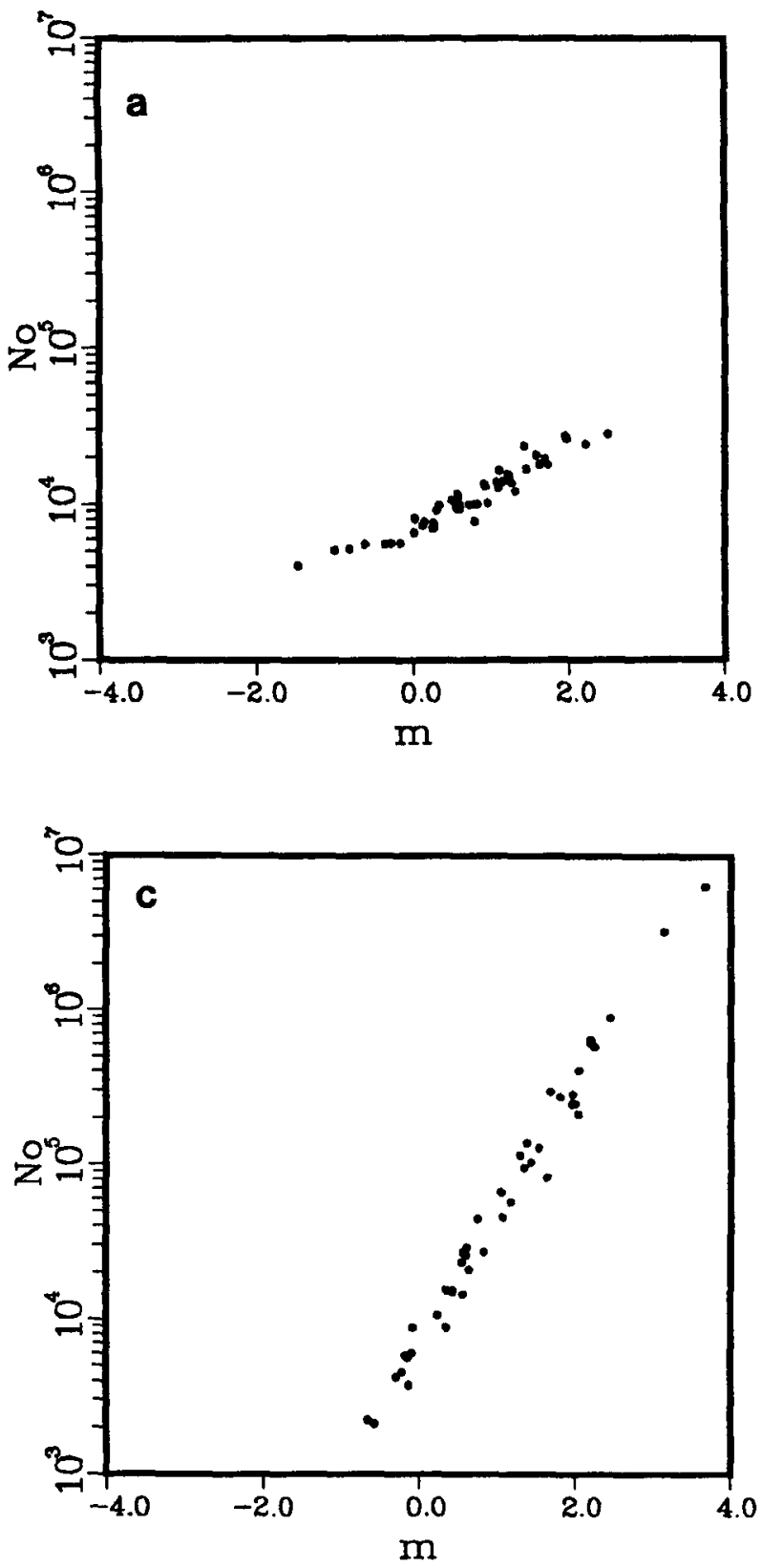

The ratio $Q=p_{\alpha, 1} / p_{\alpha, 2}$ is then

$$
Q=\frac{C_{1}}{C_{2}} \exp \left\{\left(k_{1}-k_{2}\right) m\right\}
$$

Using Ulbrich's (1983) values for $C_{1}, C_{2}, k_{1}$ and $k_{2}$ we get $Q=20 \exp (0.77 \mathrm{~m})$, where $C_{1}=10^{5.5}, C_{2}=10^{4.2}$, $k_{1}=3.57$ and $k_{2}=2.8$. The ratio $Q=p_{\alpha, 1} / p_{\alpha, 2}$ could thus be 20 for $m=0$, and 950 for $m=5$. If a mean $N_{0}-m$ relationship is assumed as $N_{0}=6 \times 10^{4}$ $\exp (3.2 m)$ as derived by Ulbrich (1983), then the ratio $Q=p_{\alpha, 1} / p_{\alpha, *}$ could vary between 5.3 (for $m=0$ ) to 33 (for $m=5$ ), while the ratio $Q=p_{\alpha, *} / p_{\alpha, 2}$ could vary

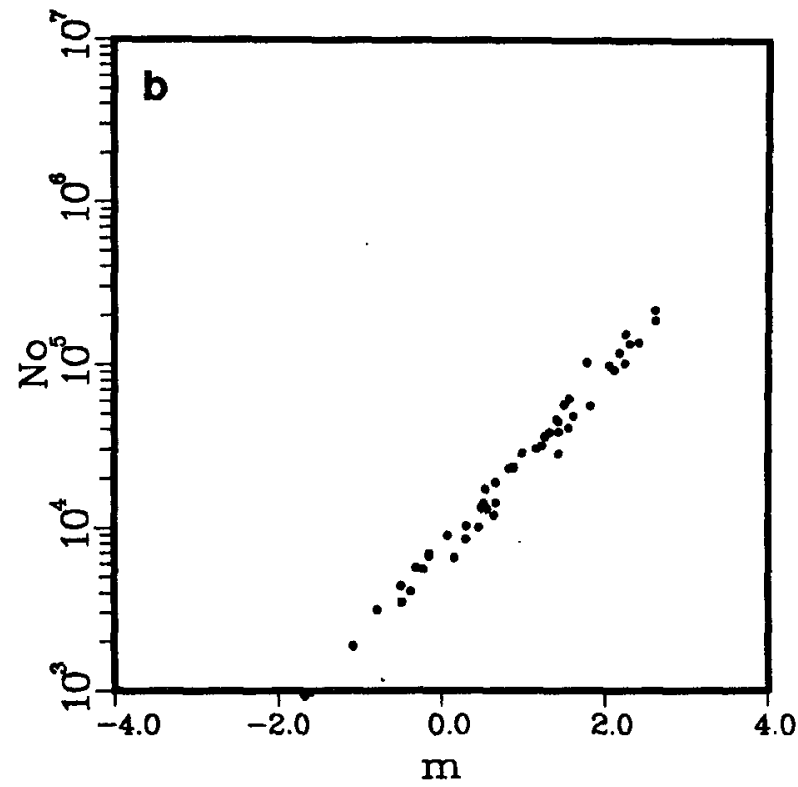

FIG. 7. (a) Scatter plot of $N_{0}$ versus $m$ estimates for average values of $N_{0}=8000 \mathrm{~mm}^{-1} \mathrm{~m}^{-3}, m=0$ and $D_{0}=2 \mathrm{~mm}$. The estimates are tightly correlated with $\ln N_{0}$ and $m$ being linearly related with slope $=0.45$. (b) As in (a) except $D_{0}=1.0 \mathrm{~mm}$. The estimates are tightly correlated with $\ln N_{0}$ and $m$ being linearly related with slope $=1.3$. (c) As in (a) except $D_{0}=0.5 \mathrm{~mm}$. The estimates are tightly correlated with $\ln N_{0}$ and $m$ being linearly related with slope $=1.8$. between 3.8 (for $m=0$ ) to 28 (for $m=5$ ). Thus small changes in the $N_{0}-m$ relations can be amplified significantly when the relations are used to estimate integral parameters such as $p_{\alpha}$.

Another interesting feature is that the region enclosed by the $N_{0}-m$ scatter is wider when plotted with $N_{0}$ in units of $\mathrm{m}^{-3} \mathrm{~mm}^{-1-m}$ as opposed to units of $\mathrm{m}^{-3}$ $\mathrm{cm}^{-1-m}$, as shown in Figs. $8 \mathrm{a}$ and $8 \mathrm{~b}$. This is because the units of $N_{0}$ depend on $m$ itself. In fact, if we plot $N_{0}$ in units of $\mathrm{m}^{-3}$ (meter) ${ }^{-1-m}$ we will practically get a straight line covering the boundary regions of $N_{0}-m$ relationships, as also recognized by Feingold and Levin (1986). 

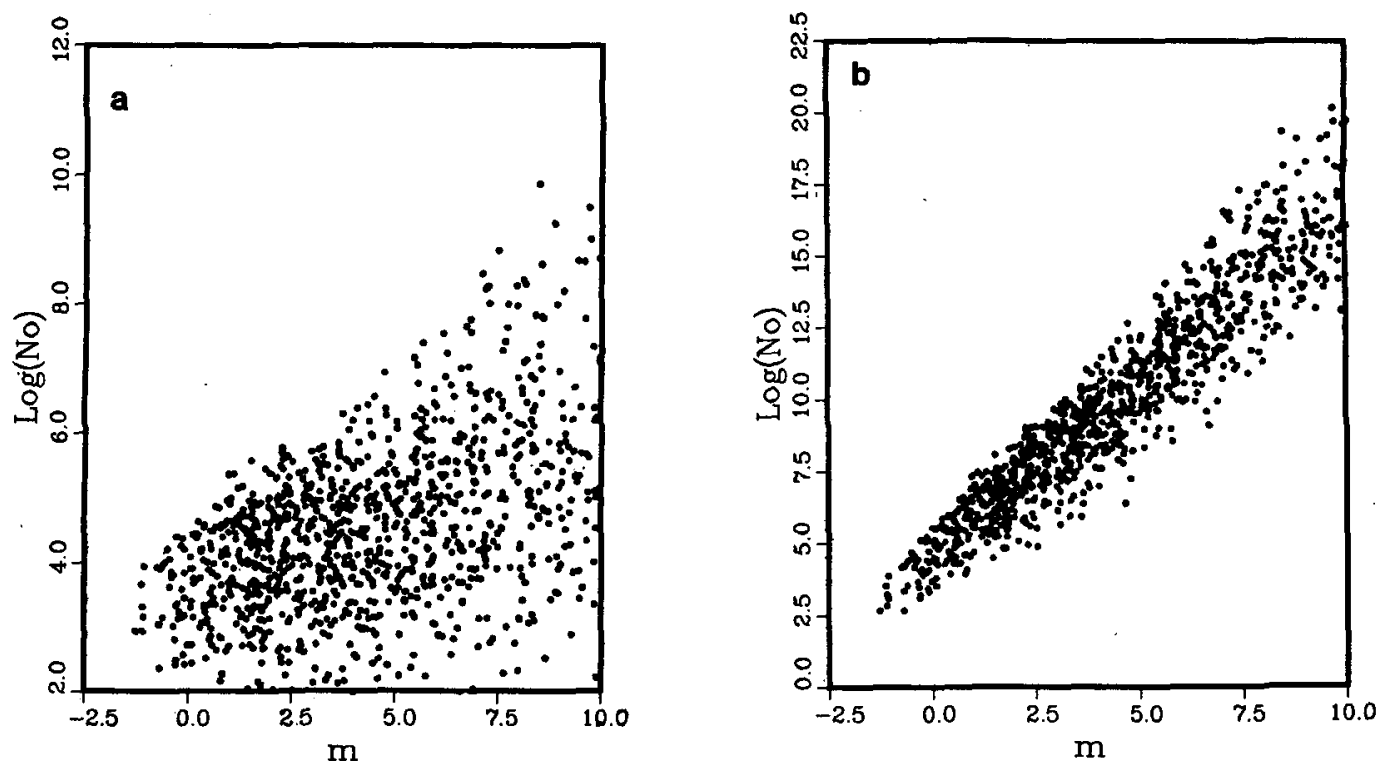

FIG. 8. (a) Global scatter plot of $N_{0}$ versus $m$ estimates where $N$ is varied between 200 to $2 \times 10^{4}\left(\mathrm{~m}^{-3} \mathrm{~mm}^{-1-m}\right)$, $D_{0}$ between 0.5 to $2.5 \mathrm{~mm}$, and $m$ between 0 and 5 . Note that the scatter plot exhibits a correlation structure between $\ln N_{0}$ and $m$. (b) As in (a) except $N_{0}$ is plotted in units of $\left(\mathrm{m}^{-3} \mathrm{~cm}^{-1-m}\right)$. Note that the scale of Fig. $8 \mathrm{~b}$ is not the same as Fig. 8a and is compressed in scale to see the full features.

Considering concentration $\left(N_{T}\right)$ as a parameter of the gamma RSD, instead of $N_{0}$, we get

$$
N_{0}=N_{T} \cdot \frac{(3.67+m)^{m+1}}{\Gamma(m+1)} \frac{1}{D_{0}^{m+1}} \text {. }
$$

For a given $D_{0}$, let us observe $N_{0}$ as a function of $m$, after expanding the gamma function using Stirling's approximation. Thus,

$$
N_{0}=N_{T}\left(1+\frac{3.67}{m}\right)^{m+1}\left(\frac{m}{2 \pi}\right)^{1 / 2} \frac{1}{D_{0}{ }^{m+1}} e^{m} .
$$

From the above equation we can see a $N_{0}-m$ relation

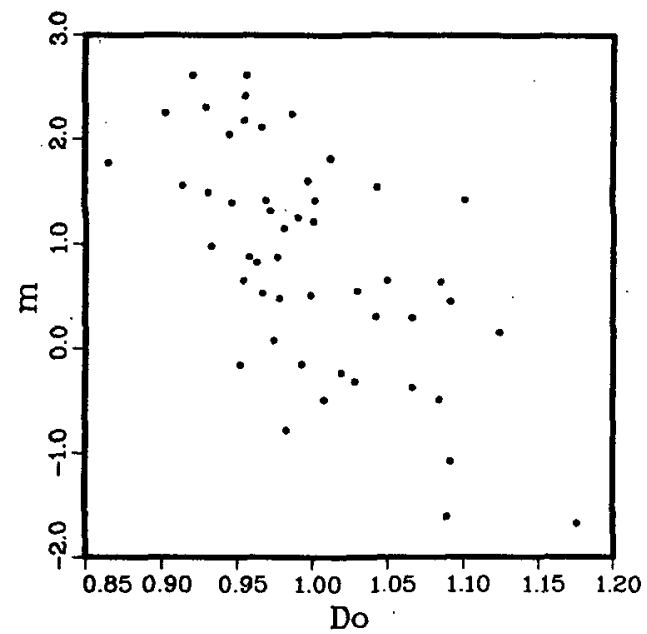

FiG. 9. Scatter plot of $\left(m, D_{0}\right)$ estimate pairs for parameters as in Fig. 7. Note the very weak correlation between the estimates. approximately of the form $N_{0}=C e^{k m}$ emerging. The reason is that the variability in $N_{T}$ is not as much as in $N_{0}$ (as observed for rainfall), and $N_{0}$ has to increase exponentially to keep reflectivity, water content, etc., within some reasonable range of values. The reason for this is that the dimension of $N_{0}$ changes with $m$.

To conclude, we have shown that the mean $N_{0}-m$ relationship derived by Ulbrich (1983) is due to the functional nature of quantities involved, and that the natural variability is made insensitive due to the strong relationship between $N_{0}$ and $m$ arising from the variable dimensionality of $N_{0}$ with $m$. The slight variability that exists in the $N_{0}-m$ relationship is sufficient to give a wide variability in quantities like $Z$ or $R$. Assuming a

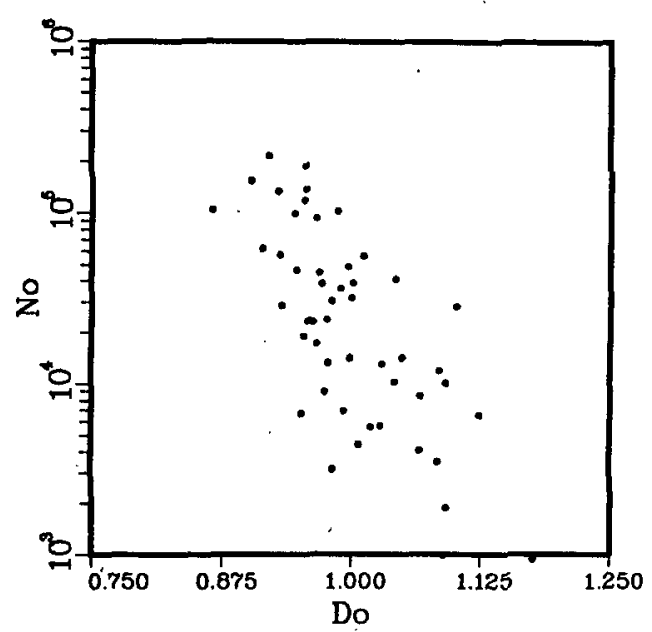

FIG. 10. As in Fig. 9 but of $\left(N_{0}, D_{0}\right)$ estimate pairs. 
mean $N_{0}-m$ relationship can cause large errors when estimating integral quantities. Hence, the three-parameter gamma RSD cannot be reduced to a two parameter form. We suggest using the concentration, $N_{T}$, rather than $N_{0}$ in the gamma RSD.

\section{Conclusions}

We have simulated two different types of measurements: viz., radar, and surface disdrometer, measurements of rainfall. These simulations involve exponential, Poisson and gamma random deviates. We also consider the effects of sample volume. The problem is computationally intensive since the parameters describing the rainfall must be varied over a wide range. Thus, we have complete control over the physical and statistical variables.

We have applied our simulations to explain why the correlation is less in plots of radar measured reflectivity versus surface measured rain intensity as compared to plots when both quantities are obtained from the same surface instrument. While physical factors are important when comparing radar measurements of rainfall aloft to surface measurements of rain intensity, it is important to have a good measure of experimental variabilities before ascribing the features to physical causes alone.

We have also applied our simulations to show that $N_{0}-m$ relations, as derived by Ulbrich (1983) using experimental disdrometer raindrop size distributions and certain higher-order moment estimators, cannot be ascribed wholly to physical causes. The simulation results indicate that the moment estimators are correlated resulting in a high degree of correlation between $N_{0}$ and $m$, even when the gamma distribution parameters were widely varied in an uncorrelated manner. We also show that assuming a mean $N_{0}-m$ relation will result in large errors when estimating reflectivity, rainfall rate, etc., since the actual $N_{0}-m$ scatter is reduced due to the units of $N_{0}$ which depend on $m$. It is preferable to use $N_{T}$ (concentration) or LWC (liquid water content) rather than $N_{0}$ in the gamma RSD.

Acknowledgments. The authors are grateful to Dr. Hari Iyer of Colorado State University for many helpful discussions and for a critical reading of this paper. This work was supported by the U.S. Army Research Office via NCAR Subcontract S3024 and via a grant to Colorado State University, DAAL03-86-K-0117. One of the authors (VC) is a Fellow of CSU's Institute of Computational Studies which provided Cyber 205 computer resources for this study through NASA Grant NGT 06-002-802.

\section{APPENDIX A}

\section{Correlation for Varying RSD Parameters}

Let us consider a sample volume with $f_{1}$ fraction filled with RSD1 with parameters $\left(N_{0}{ }^{1}, D_{0}{ }^{1}, m^{1}\right)$ and $f_{2}$ fraction filled with RSD2 with parameter $\left(N_{0}^{2}, D_{0}{ }^{2}\right.$, $\left.m^{2}\right) \ldots$ and $f_{k}$ portion filled with RSD $k$ with parameters $\left(N_{0}{ }^{k}, D_{0}{ }^{k}, m^{k}\right)$, such that $f_{1}+f_{2} \cdots f_{k}=1$. If $n$ is the total number of scatterers in the volume then it is composed of $n_{1}, n_{2} \ldots n_{k}$ scatterers each from the respective RSD.

We need to compute the correlation between parameters $\hat{p}_{\alpha}, \hat{p}_{\beta}$ computed for the volume defined by Eq. (8d)

$$
\begin{aligned}
\operatorname{cov}\left[\hat{p}_{\alpha}, \hat{p}_{\beta}\right]= & \frac{C_{\alpha} C_{\beta}}{V^{2}} \operatorname{cov}\left[\sum_{i=1}^{n} D_{i}^{\alpha}, \sum_{i=1}^{n} D_{i}^{\beta}\right] \\
= & \frac{C_{\alpha} C_{\beta}}{V^{2}} \operatorname{cov}\left[\sum_{i=1}^{n_{1}}\left(D_{i}^{1}\right)^{\alpha}+\sum_{i=1}^{n_{2}}\left(D_{i}^{2}\right)^{\alpha}\right. \\
& +\cdots \sum_{i=1}^{n_{k}}\left(D_{i}^{k}\right)^{\alpha}, \sum_{i=1}^{n_{1}}\left(D_{i}^{1}\right)^{\beta} \\
& \left.+\sum_{i=1}^{n_{2}}\left(D_{i}^{2}\right)^{\beta}+\cdots \sum_{i=1}^{n_{k}}\left(D_{i}^{k}\right)^{\beta}\right] \\
= & \frac{C_{\alpha} C_{\beta}}{V^{2}} \sum_{j=1}^{k} \operatorname{cov}\left[\sum_{i=1}^{n_{j}}\left(D_{i}^{j}\right)^{\alpha}, \sum_{i=1}^{n_{j}}\left(D_{i}^{j}\right)^{\beta}\right] .
\end{aligned}
$$

Using Eq. (14) the above equation can be simplified to,

$$
=\frac{C_{\alpha} C_{\beta}}{V^{2}}\left[\sum_{j=1}^{k} E\left(n_{j}\right) E^{j}\left[D^{\alpha+\beta}\right]\right],
$$

$E^{j}$ means expectation under $j$ th RSD and it can be easily shown that since $f_{1} \ldots f_{k}$ is a mutually exclusive partition and $n$ is distributed Poisson, $n_{j}$ is also distributed Poisson with mean $f_{j} \epsilon(n)$ (Mood et al., 1974).

From Eq. (11a)

$$
\operatorname{var}\left[\hat{p}_{\alpha}\right]=\frac{C^{2}}{V^{2}} \operatorname{var}\left[\sum_{i=1}^{n} D_{i}^{\alpha}\right]
$$

and this can be written using similar arguments as for covariance as

$$
=\frac{C_{\alpha}^{2}}{V^{2}} \sum_{j=1}^{k}\left\{\operatorname{var}\left[\sum_{i=1}^{n_{j}}\left(D_{i}^{j}\right)^{\alpha}\right]\right\}
$$

Using (11b) we write this as

$$
=\frac{C_{\alpha}{ }^{2}}{V} \sum_{j=1}^{k}\left[N_{\sigma}{ }^{j} \frac{\Gamma\left(m^{j}+2 \alpha+1\right)}{\Lambda_{j}^{\left(m^{j}+2 \alpha+1\right)}}\right] .
$$

Using (A3) and (A5) we can write the correlation as $\rho$

$$
=\frac{\sum_{j=1}^{k} N_{\sigma^{j}} \frac{\Gamma\left(m^{j}+\alpha+\beta+1\right)}{\Lambda_{j}^{\left(m^{j}+\alpha+\beta+1\right)}}}{\left[\sum_{j=1}^{k} N_{\sigma^{j}} \frac{\Gamma\left(m^{j}+2 \alpha+1\right)}{\Lambda_{j}^{\left(m^{j}+2 \alpha+1\right)}}\right]^{1 / 2}\left[\sum_{j=1}^{k} N_{0}{ }^{j} \frac{\Gamma\left(m^{j}+2 \beta+1\right)}{\Lambda_{j}^{\left(m^{j}+2 \beta+1\right)}}\right]^{1 / 2}} .
$$

If $m$ does not vary in the sample volume Eq. (A6) reduces to 


$$
\rho=\rho^{\prime} \frac{\sum_{j=1}^{k} N_{0}^{j}\left(D_{0}^{j}\right)^{(m+\alpha+\beta+1)}}{\left[\sum_{j=1}^{k} N_{0}^{j}\left(D_{0}^{j}\right)^{(m+2 \alpha+1)}\right]^{1 / 2}\left[\sum_{j=1}^{k} N_{\sigma^{j}}\left(D_{0}^{j}\right)^{(m+2 \beta+1)}\right]^{1 / 2}},
$$

where

$$
\rho^{\prime}=\frac{\Gamma(m+\alpha+\beta+1)}{\Gamma(m+2 \alpha+1) \Gamma(m+2 \beta+1)} .
$$

If $D_{0}$ is also same and only if $N_{0}$ changes thereby indicating the shape of RSD is same but mean values are different we can see that $\rho$ reduces to Eq. (16).

Example: Let $k=2$, and let the RSD parameters be as follows:

$$
\begin{aligned}
N_{0}{ }^{1} & =8000 & N_{0}^{2} & =10000 \\
D_{0}{ }^{1} & =2.0 & D_{0}{ }^{2} & =1.5 \\
m^{1} & =1 & m^{2} & =1.5 .
\end{aligned}
$$

Let $\alpha, \beta$ be 3.67 and 6 corresponding to rainfall rate and $Z$, respectively, then $\rho=\rho^{\prime}(0.988)$. Due to the nature of the expressions $\rho$ will be close to the RSD which has the largest $D_{0}$ value and we would expect $\rho$ to lie between the values of correlation shown in Fig. 2. Since the variability in correlation between $R$ and $Z$ as RSD parameters is very small, this small perturbation in $\rho$ from $\rho^{\prime}$ is not surprising.

The above example shows that the tight correlation between disdrometer estimates of rainfall rate and reflectivity is not reduced when we have mixtures of RSDs in the sample volume. It can be easily shown that the spread between disdrometer-measured $R$ and radar-measured $Z$ increases in the case of mixtures as compared to single RSD case.

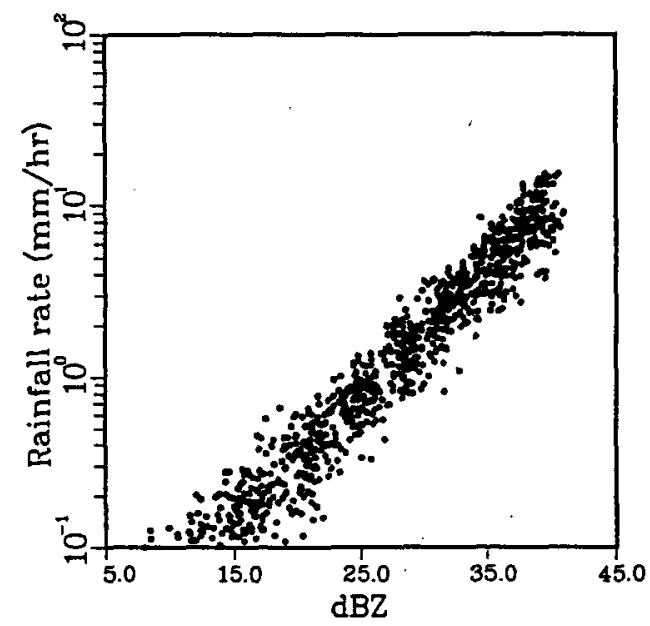

FIG. A1. Comprehensive scatterplot of rainfall rate versus $\mathrm{dBZ}$, both of which are derived from mixture of drop size spectra where the mixture proportion is varied randomly. The parameters of the constituent RSDs are kept close so as not to vary their properties widely. Sample volume is $1.8 \mathrm{~m}^{3}$.

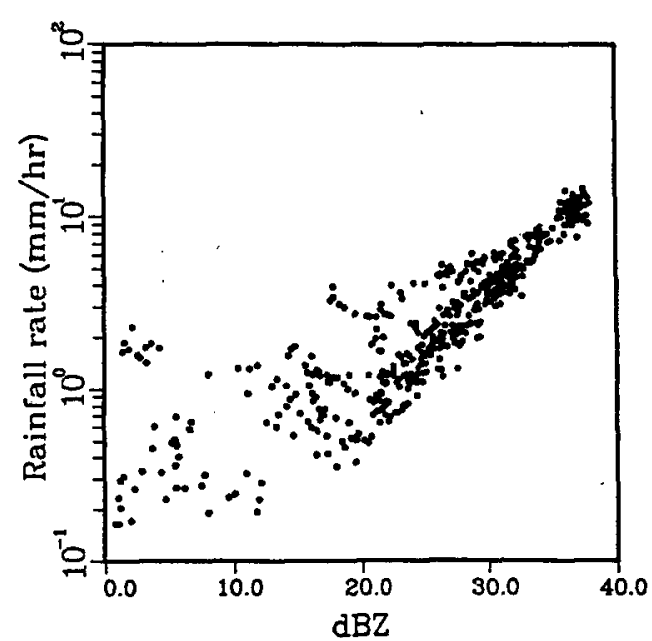

FIG. A2. As in Fig. A1 with $\mathrm{dBZ}$ "measured" by radar except the mixing proportion of the constituent RSDs has been perturbed from those of Fig. Al according to the description in appendix A.

Differences between radar and disdrometer/PMS probe measurements can be introduced when the fractional contribution of the individual RSDs into a mixture are not matched. In the case of practical experiments this can happen in many ways and therefore we present here a simple model to study this effect.

Since aircraft-mounted PMS probes sample RSDs along a linear path whereas radar samples over an area (dropping the differences in vertical dimension for simplicity) the fractional contribution of the individual RSDs to the mixture must be weighted according to $L$ and $L^{2}$ for probe and radar, respectively, $L$ being a linear dimensional factor. Similar arguments could be used to describe the advection of rain cells over a surface disdrometer. Figures A1, A2 show the simulation results for a collective sample volume of $1.8 \mathrm{~m}^{3}$. Each data point consists of a mixture from two mean RSDs, viz., $N_{0}, D_{0}, m$ and $5 N_{0}, D_{0}+0.1 \mathrm{~mm}, m+0.25$. To generate the figure each mean $N_{0}, D_{0}, m$ is varied over a range normally found in rainfall. The mixing ratio $f$ is varied randomly for each mean $N_{0}, D_{0}, m$. The relative contribution for the two mean RSDs for the disdrometer is $\{f: 1-f\}$ whereas for radar it is $\left\{f^{2}:(1\right.$ $\left.-f)^{2}\right\}$ which is normalized by the factor $f^{2}+(1-f)^{2}$. It is clear that the scatter in Fig. A2 is considerably more than in Fig. A1, which is due to the combined effect of mixing RSDs according to linear and areal weighting.

\section{APPENDIX B}

\section{The Gamma Distribution Function}

The standard gamma has the following distribution function:

$$
f(x)=\frac{1}{\Gamma(x)} x^{\alpha-1} e^{-x}
$$


Using Cheng's notation, let

$$
M=\max _{x}\left\{\frac{f(x)}{g(x)}\right\}
$$

be finite. Take a pair of independent $u(0,1)$ variables $U_{1}$ and $U_{2}$ say. Let $x=G^{-1}\left(U_{1}\right)$. Then if $[f(x)] /[M g(x)]$ $\geqslant U_{2}$ accept $x$, otherwise reject it. Each accepted $x$ has density $f(x)$. Cheng suggests using $f(x)$ same as (B1) and

where

$$
g(x)=\frac{\lambda \mu x^{\lambda-1}}{\left(\mu+x^{\lambda}\right)^{2}}
$$

$$
\mu=\alpha^{\lambda} \text { and } \lambda=\sqrt{2 \alpha-1} .
$$

$M$, the expected number of trials varies between 1.47 and 1.13 as varies from 1 to $\infty$. The advantage of this method is it gives a reasonably small rejection ratio which translates into starting vector length of uniform deviates not much greater than the length of string of gamma required. This method also has one decision taking spot which can be easily accommodated by using control bit vectors that would have bit value " 1 " for accepted elements of the vector that can be gathered later. The following steps show equivalent vector form for Cheng's algorithm. (We denote vectors with an arrow above the symbols.)

Step 1: Generate a pair of uniform random vectors $\mathbf{U}$ and $\mathbf{U}_{2}$

Step 2: Set $\mathbf{V}=a \log \left\{\mathbf{U}_{1} /\left(1-\mathbf{U}_{1}\right)\right\}$, $\mathbf{X}=e^{\mathbf{v}}$

Step 3: If $b+c \mathbf{V}-\mathbf{X} \geqslant \log \left[\mathbf{U}_{1} \cdot \mathbf{U}_{1} \cdot \mathbf{V}_{2}\right]$ set bit vector elements to 1 .

The constants are:

and

$$
a=(2 \alpha-1)^{-1 / 2}, \quad b=\alpha-\log 4
$$

$$
c=\alpha+a^{-1} \text {. }
$$

Note that in the above equations, arithmetic operations like multiplication, division and exponentiation imply element by element operation. The length of these vectors $n^{\prime}$, is $M n$ where $n$ is the vector length of random deviates required. If the number of " 1 " bits fall short of $n^{\prime}$ then those few random deviates can be computed by scalar version of algorithm. Timing runs made on Cyber 205 indicate that length 100 deviates can be computed 2.95 times faster, whereas the speedup increases to 6.1 for length 50 and 7.1 for length 2500 .

\section{APPENDIX C}

\section{Correlation for Variable Sampling Volume}

Let the sampling volume of drops vary as $V(D)$ $=V_{0} D^{b}$. Then it can be easily shown that if drop size distribution is described by gamma with parameters $N_{0}, \Lambda, m$ the sampled distribution is also gamma with parameters $N_{0}, \Lambda,(m+b)$. Moment estimates like $Z$,
$R$ have to be adjusted for sample volume to get unbiased estimates so the new estimates for $p_{\alpha}$ become

$$
\hat{p}_{\alpha}=C_{\alpha} \sum_{i=1}^{n} \frac{D_{i}^{\alpha}}{V_{i}}=\frac{C_{\alpha}}{V_{0}} \sum_{i=1}^{n} D_{i}^{(\alpha-b)}
$$

Viewing this estimator described by $\mathrm{C} 1$ as $(\alpha-b)$ th moment of gamma with parameters $N_{0}, \Lambda, m+b$ we can show using (9) and (10) that $\hat{p}_{\alpha}$ is an unbiased estimator of $p_{\alpha}$ and fractional standard deviation (FSD) of $\hat{p}_{\alpha}$ is obtained from Eq. (11) as

$$
\operatorname{FSD}\left(\hat{p}_{\alpha}\right)=\left[\frac{\Lambda^{m+b+1}}{N_{0} V}\right]^{1 / 2} \cdot \frac{\Gamma^{1 / 2}(m+2 \alpha-b+1)}{\Gamma(m+\alpha+1)} .
$$

Similarly, using Eqs. (13), (14) and (15) we can show that the correlation coefficient $\rho_{\alpha, \beta}$, the correlation coefficient for the estimators $\hat{p}_{\alpha}$ and $\hat{p}_{\beta}$ is given by

$$
\rho_{\alpha, \beta}=\frac{\Gamma(m+\alpha+\beta-b+1)}{\left\{\Gamma(m+2 \alpha-b+1) \Gamma(m+2 \beta-b+1)^{1 / 2}\right.} .
$$

The following example shows that the correlation coefficient between estimates of $Z$ and $R$ is not changed much when we include the effect of change in sample volume with size.

Example: Let $\alpha, \beta$ be 6 and 3.67 corresponding to estimates of $Z$ and $R$. Let $b$ be 0.5 (from Gertzman and Atlas, 1977). When we assume the DSD is gamma with $m=2$ then the correlation coefficient between these parameter estimates changes from 0.799 to 0.791 as we include the effect of sample volume variability with size of the drop.

\section{REFERENCES}

AGU, 1984: A new interdisciplinary focus on precipitation research, committee on precipitation. Eos, 65, 377.

Atlas, D., 1964: Advances in radar meteorology. Adv. in Geophys., $10,317-478$.

- , and C. W. Ulbrich, 1977: Path- and area-integrated rainfall measurement by microwave attenuation in the $1-3 \mathrm{~cm}$ band. J. Appl. Meteor., 16, 1322-1331.

Bringi, V. N., T. A. Seliga and E. A. Mueller, 1982: First comparisons of rainfall rates derived from radar differential reflectivity and disdrometer measurements. IEEE Trans. Geosci. Remote Sensing, Vol. GE-20(2), 201-204.

Cheng, R. C. H., 1978: Generating of gamma variables with nonintegral shape parameter. Appl. Stat., 26, 71-75.

Cornford, S. G., 1967: Sampling errors in measurements of raindrop and cloud droplet concentrations. Meteor. Mag., 96, 271-282.

Doviak, R. J., and D. S. Zrnić, 1984: Doppler Radar and Weather Observations. Academic Press.

Feingold, G., and Z. Levin, 1986: The Lognormal fit to raindrop spectra from frontal convective clouds in Israel. Accepted for publication in J. Climate Appl. Meteor. (in press).

Gertzman, H. S., and D. Atlas, 1977: Sampling errors in the measurement of rain and hail parameters. J. Geophys. Res., 82(31), 4955-4966.

Goddard, J. W. F., and S. M. Cherry, 1984: Quantitative precipitation measurements with dual linear polarization radar. Proc. 22nd Conf. on Radar Meteorology, Zurich, Amer. Meteor. Soc., 352357.

, - and V. N. Bringi, 1982: Comparison of dual-polarization measurements of rain with ground-based disdrometer measurements. J. Appl. Meteor., 21, 252-256. 
Gordan, G. L., and J. D. Marwitz, 1984: An airborne comparison of three PMS probes. J. Atmos. Oceanic Technol., 1, 22-27.

Jameson, A. R., 1985: Microphysical interpretation of multiparameter radar measurements in rain-Part III: Interpretation and measurement of propagation differential phase shift between orthogonal linear polarizations. J. Atmos. Sci., 42, 607-614.

Joss, J., and A. Waldvogel, 1967: Ein Spektograph fur Niederschlagstrophen mit automatischer Auswertung. Pure Appl. Geophys., 68, 240-246.

- , and - 1969: Raindrop size distribution and sampling size errors. J. Atmos. Sci., 26, 566-569.

- , and E. G. Gori, 1978: Shapes of raindrop size distributions. $J$. Appl. Meteor., 17, 1054-1061.

Kennedy, W. J., Jr., and J. E. Gentle, 1980: Statistical computing, Vol. 33, Statistics: Textbooks and Monographs, Marcel Dekker.

Mielke, P. W., Jr., 1976: Simple iterative procedures for two parameter gamma distribution maximum likelihood estimates. J. Appl. Meteor., 15, 181-183.

Mood, M. A., F. A. Graybill and D. C. Boes, 1974: Introduction to the Theory of Statistics, McGraw-Hill.

Mueller, E. A., 1984: Calculations procedure for differential propagation phase shift. Preprints, 22nd Conf. on Radar Meteorology, Zurich, Amer. Meteor. Soc., 397-399.

Richards, W., and C. Crozier, 1983: Precipitation measurements with a C-band radar in Southern Ontario. Atmos.-Ocean, 21, 125 137.

Sachidanda, M., and D. S. Zrnić, 1986: Differential propagation phase shift and rainfall rate estimation. Radio Sci., 21, 235-247.

Sasyo, Y., 1965: On the probabalistic analysis of precipitation par- ticles. Proceedings of the Int. Conf. on Cloud Physics, Int. Assoc. Meteor. Atmos. Phys. 254-259.

Seliga, T. A., and V. N. Bringi, 1976: Potential use of radar differential reflectivity measurements at orthogonal polarizations for measuring precipitation $J$. Appl. Meteor., 15, 69-76.

$\longrightarrow$, and - 1978: Differential reflectivity and differential phase shift: Applications in radar meteorology. Radio Sci., 13(2), 271275 .

- K. K. Aydin and H. Direskeneli, 1986: Disdrometer measurements during an intense rainfall event in Central Illinois: Implication for differential reflectivity radar observation. J. Climate Appl. Meteor. (in press).

Semplak, R. A., and R. H. Turrin, 1969: Some measurements of attenuation by rainfall at $18.5 \mathrm{GHz}$. Bell Syst. Tech. J., 48(6), 1757-1787.

Ulbrich, C. W., 1983: Natural variations in the analytical form of raindrop size distribution. J. Climate Appl. Meteor., 22, 17641775.

- , and D. Atlas, 1985: Extinction of visible and infrared radiation in rain: Comparison of theory and experiment. J. Atmos. Oceanic Technol., 2, 331-339.

Wong, R. K. W., and N. Chidambaram, 1985: Gamma size distribution and stochastic sampling errors. J. Climate Appl. Meteor., 24, 568-579.

Zawadzki, I., 1984: Factors affecting the precision of radar measurements of rain. Preprints, 22nd Conf. on Radar Meteorology, Zurich, Amer. Meteor. Soc.

Zrnić, D. S., 1975: Simulation of weatherlike Doppler spectra and signals. J. Appl. Meteor., 14, 619-620. 